\title{
YIELD PERFORMANCES AND CUP QUALITY OF SOME BTRI TEST CLONES OF TEA
}

\section{M.I. Hossain 1*, M.J . Dutta², A. Q. Khan ${ }^{3}$, M.S.A. Mamun ${ }^{4}$ and M.M. Rana5}

Received 13 September 2011, Revised 3 November 2011, Accepted 25 December 2011, Published online 31 December 2011

\begin{abstract}
A long term experiment was conducted to investigate the yield and quality performances of four vegetative propagated test clones of tea coded as A/8/01, A/17/22, $\mathrm{A} / 22 / 27$ and A/22/40 at Bangladesh Tea Research Institute (BTRI) farm during 1996-2010. A standard clone BT1 was considered as control. Cuttings of the test clones were collected from the selected bushes of Amo tea estates and were raised at BTRI nursery. Then saplings were put to long term yield and quality trial following Latin Square Design (LSD) with 3 replications. The green leaf was harvested at weekly interval during the plucking season starting from mid March to mid December throughout the experimental period. Yield data were recorded and analyzed statistically using MSTAT programme. Results of the experiment revealed that among the test clones A/22/40 gave the highest significant yield of $3509.1 \mathrm{~kg} \mathrm{ha}^{-1}$ of made tea followed by BT1 (3203.69 $\mathrm{kg} \mathrm{ha}^{-1}$ ), A/8/01 (2912.24 kg ha-1), A/17/ 22 (2817.76 kg ha-1) and $\mathrm{A} / 22 / 27$ (2278.78 $\left.\mathrm{kg} \mathrm{ha}^{-1}\right)$ from the average of 9 years (2002-2010) at mature stage. At immature stage i.e. $1^{\text {st }}$ year to $5^{\text {th }}$ year after plantation yield difference was insignificant. The overall cup quality of the test clones was assessed by conventional organoleptic test. The cup quality of $\mathrm{A} / 8 / 01, \mathrm{~A} / 17 / 22, \mathrm{~A} / 22 / 40$ as well as the standard clone BT1 was found to be above average (AA) while the cup quality of $\mathrm{A} / 22 / 27$ was average (A). Considering the yield and quality potentials, the test clone $\mathrm{A} / 22 / 40$ has appeared quite promising to be released as a standard clone.
\end{abstract}

Keywords: Yield Performances, Cup Quality, Test Clones, BTRI

${ }^{1}$ Principal Scientific Officer (in-charge), Botany Division, Bangladesh Tea Research Institute, Srimangal, Moulvibazar, Bangladesh; ${ }^{2}$ Ex. Director, Bangladesh Tea Research Institute, Srimangal, Moulvibazar, ${ }^{3}$ Seniro Scientific Officer, Soil Science Division, Bangladesh Tea Research Institute, Srimangal, Moulvibazar, Bangladesh; ${ }^{4}$ Scientific Officer, Entomology Division, Bangladesh Tea Research Institute, Srimangal, Moulvibazar, Bangladesh; ${ }^{5}$ Scientific Officer, Agronomy Division, Bangladesh Tea Research Institute, Srimangal, Moulvibazar, Bangladesh

*Corresponding author's email: ismailbtri@yahoo.com

Reviewed by Prof. Dr. A.K.M. Golam Sarwar, Bangladesh Agricultural University, Mymensingh, Bangladesh

\section{Introduction}

Tea is one of the most important cash crops in Bangladesh and south Asia. It is also an important food commodity of international trade. The principal types of tea produced and consumed in the world are black and green tea with small amounts of other types. The world tea production is 4,162 million $\mathrm{kg}$ in 2010 (ITC, 2011). It is a major cash crop as well as a significant export item of Bangladesh accounting for about $0.81 \%$ of the GDP (BTB, 2002).

Our present yield hectare-1 is quite low compared to other tea growing countries of the world. Because a large portion of our tea growing area is covered with seedling plants which are over 60 years old and are of unimproved jats of lower productivity. Moreover, a significant area of this old plantation has low plant population density resulting in lower productivity and poor quality of tea. The increasing cost of production as well as adverse climatic conditions has led to marginal economic return to the tea industry. In these circumstances, the industry needs to replant and extend new tea areas with improved planting materials of higher yield and good quality. Due to the heterogenous nature of tea seedlings, the available seed except biclonal stocks, could not guarantee the production of improved planting material (Njuguna, 1990). Therefore, emphasis should be given on selection and planting of vegetatively propagated material i.e. high yielding quality clones for better yield and quality of tea (Dutta and Alam, 2001). 
Clonal selection is the most popular practice in tea for evolving better varieties. Desirable potential plants are isolated from the seedling population that have derived from superior jats or breeding lines. Procedure of clonal selection is more or less same in all the tea growing countries (Tubbs, 1946; Vissar \& Kehl, 1958; Wight, 1961; Barua, 1964).

With an objective of evolving planting materials with high yield and quality potential, BTRI has put its priorities of research on clonal selection and hybridization programme since its inception. The clonal selection programme was initiated in 1959 and hybridization programme in 1965 (Rashid and Alam, 1990). As an outcome of these works, the institute so far has released eighteen vegetative clones in the Bangladesh tea (BT) series (BT1 - BT18) to the industry.

Few more test clones are in various stages of trial. The present experiment was carried out to study the long term yield and quality performances of four vegetatively propagated test clones of tea selected from the Amo tea estate.

\section{Materials and Methods}

The experiment was carried out with four test clones and one standard clone of tea in the BTRI experimental farm, during the period from April, 1996 to December, 2010. Cuttings were collected from the selected bushes of Amo tea estate during 1994, which were raised at BTRI nursery. After the rooting trial in nursery, the selected test clones, namely A/8/01, A/17/22, A/22/27 and A/22/40 were put to long term yield and quality trial in 1996. The experiment was laid out in Latin Square Design (LSD) with three replications. Each plot was $5 \mathrm{~m} \mathrm{x} 5 \mathrm{~m}$ in size with $105 \mathrm{~cm}$ (row-row) x $60 \mathrm{~cm}$ (plant-plant) spacing. There were 25 plants per plot. A standard clone BT1 was used as control for yield and quality comparison. The experiment was conducted in rain-fed condition. Yield data was collected during the cropping seasons throughout the experimental period.

The N-P-K mixture was applied at young and mature tea as per BTRI recommendations (Kibria and Rashid, 1994; Kibria and Uddin, 1998). Pruning was followed for young tea: Decentre - Prune - Skiff - Prune - Skiff (Shahiduzzaman et al., 2002) and for mature tea: Light Prune (LP) - Deep skiff (DSK) Medium skiff (MSK) - Light skiff (LSK) cycle as per BTRI recommendations (Rashid, 1986). The green leaf was harvested at weekly interval during the plucking season starting from mid March to mid December throughout the experimental period. Yield data were recorded and analyzed statistically using MSTAT programme in a microcomputer. The mean values were adjudged by DMRT. The yield was expressed as mean yield of green leaf $g$ plant ${ }^{-1}$ and is presented separately for immature (1st - 5th year) and mature ( $6^{\text {th }}-14^{\text {th }}$ year) stage. The made tea ( $\mathrm{kg} \mathrm{ha}^{-1}$ ) was also calculated on the basis of 23\% recovery from green leaf and 15875 plants $\mathrm{ha}^{-1}$ at $105 \mathrm{~cm}$ x $60 \mathrm{~cm}$ spacing. The quality performances of manufactured by CTC method in the BTRI mini tea factory. Quality of all the test clones and control were assessed weekly by conventional organoleptic test and scored numerically. General characteristics of four test clones and control BT1 is given in Table 1.

Table 1. General characteristics of some selected test clones and BT1

\begin{tabular}{|c|c|c|c|c|c|c|}
\hline Clone & Bush characters & Leaf type & $\begin{array}{l}\text { Pruning } \\
\text { recovery }\end{array}$ & $\begin{array}{l}\text { Nursery } \\
\text { rooting }\end{array}$ & $\begin{array}{c}\text { Cup } \\
\text { quality }\end{array}$ & $\begin{array}{c}\text { Manufacturing } \\
\text { Preference }\end{array}$ \\
\hline $\mathrm{T} 1-\mathrm{A} / 8 / 01$ & $\begin{array}{l}\text { Assam, vigorous, ortho- } \\
\text { plagiotropic, good girth, } \\
\text { fairly compact }\end{array}$ & $\begin{array}{l}\text { Large, light green } \\
\text { with prominent } \\
\text { pointed apex, semi } \\
\text { erect }\end{array}$ & Good & Good & $\begin{array}{l}\text { Above } \\
\text { average }\end{array}$ & CTC \\
\hline T2-A/ 17/ 22 & $\begin{array}{l}\text { Assam hybrid, medium } \\
\text { bush, vigorous, Ortho- } \\
\text { plagiotropic, grower } \\
\text { with good spread, quite } \\
\text { compact plucking table }\end{array}$ & $\begin{array}{l}\text { Medium to large, } \\
\text { semi dark green, } \\
\text { green, semi-erect, } \\
\text { pointed apex }\end{array}$ & Good & Good & $\begin{array}{l}\text { Above } \\
\text { average }\end{array}$ & СТC \\
\hline T3-A/22/ 27 & $\begin{array}{l}\text { Medium bush, heavy } \\
\text { girth, good grower with } \\
\text { good spread, } \\
\text { orthotropic, thick } \\
\text { shoots }\end{array}$ & $\begin{array}{l}\text { Medium to large, } \\
\text { light green, erect, } \\
\text { prominent leaf } \\
\text { apex }\end{array}$ & Good & Good & Average & СТC \\
\hline T4- A/ 22/40 & $\begin{array}{l}\text { Assam hybrid, medium } \\
\text { bush, vigorous } \\
\text { orthotropic grower with } \\
\text { a very good spread } \\
\text { forming dense plucking } \\
\text { table }\end{array}$ & $\begin{array}{l}\text { Medium to large, } \\
\text { semi-dark green, } \\
\text { glossy, semi-erect, } \\
\text { serrated margin }\end{array}$ & Good & Good & $\begin{array}{l}\text { Above } \\
\text { average }\end{array}$ & СТC \\
\hline $\begin{array}{l}\text { T5-BT1 } \\
\text { (Control) }\end{array}$ & $\begin{array}{l}\text { Bush size medium, } \\
\text { plagiotropic, thickly } \\
\text { branched and compact, } \\
\text { fairly dense and evenly } \\
\text { distributed plucking } \\
\text { points with short } \\
\text { internodes }\end{array}$ & $\begin{array}{l}\text { Leaves semi-dark } \\
\text { green, medium } \\
\text { sized, horizontal } \\
\text { pose. Prominent } \\
\text { long apex, leaf } \\
\text { margin deeply } \\
\text { serrated, leaf blade } \\
\text { wavy }\end{array}$ & Good & Good & $\begin{array}{l}\text { Above } \\
\text { average }\end{array}$ & CTC \\
\hline
\end{tabular}


The tea clones were categorized into the following classification

\begin{tabular}{lccc}
\hline $\begin{array}{l}\text { Category of } \\
\text { clones }\end{array}$ & $\begin{array}{c}\text { Yield } \\
\text { clone } \\
\left(\mathrm{kg} \mathrm{ha}^{-1}\right)\end{array}$ & $\begin{array}{c}\text { Standard } \\
\text { clone } \\
\left(\mathrm{kg} \mathrm{ha}^{-1}\right)\end{array}$ & $\begin{array}{c}\text { Quality } \\
\text { clone } \\
\left(\mathrm{kgha}^{-1}\right)\end{array}$ \\
\hline $\begin{array}{l}\text { Yield } \\
\text { Performance }\end{array}$ & $>4000$ & $\begin{array}{c}3000- \\
4000\end{array}$ & $<3000$ \\
Cup Quality* & AA or A & AA & E
\end{tabular}

* Quality score:

$\mathrm{E}=$ Excellent $(34$ to $>34$ out of 50 )

AA = above average (32 to $<34$ out of 50 )

$A=$ average (30-32 out of 50 )

$\mathrm{BA}=$ Below Average $(<30$ out of 50$)$

\section{Results and Discussion}

The mean yield of green leaf ( $\mathrm{g}$ plant $\left.{ }^{-1}\right)$ at immature (1 $1^{\text {st }}-5^{\text {th }}$ year) and mature $\left(6^{\text {th }}-14^{\text {th }}\right.$ year) stage is presented in Table 2 and Table 3 , respectively. At the initial stage of growth, all test clones showed similar yield trend as control (BT1) (Table 2). Green leaf yield was converted as made tea $\left(\mathrm{kg} \mathrm{ha}^{-1}\right)$ over the experimental period. The mean made tea production at immature and mature stage is presented in Table 4 and Table 5, respectively. During the experimental period, test clone A/22/40 maintained higher trend of yield over control whereas A/8/01, A/ 17/22, A/22/27 maintained lower trend of yield over control (Table 5).

Table-2. Mean yield of green leaf ( $g$ plant ${ }^{-1}$ ) at immature stage ( $1^{\text {st }} 5^{\text {th }}$ year)

\begin{tabular}{lcccccc}
\hline \multicolumn{1}{c}{ Year } & $1^{\text {st }}$ Year Skiff & $2^{\text {nd }}$ Year Prune & $3^{\text {rd }}$ Year Skiff & $4^{\text {th }}$ Year Prune & $5^{\text {th }}$ Year Skiff & Average \\
Clone & 1997 & 1998 & 1999 & 2000 & 2001 & \\
\hline A/8/01 & $63.39 \mathrm{a}$ & $156.50 \mathrm{a}$ & $316.83 \mathrm{a}$ & $395.33 \mathrm{a}$ & $716.04 \mathrm{~b}$ & $329.62 \mathrm{a}$ \\
$\mathrm{A} / 17 / 22$ & $57.94 \mathrm{a}$ & $169.00 \mathrm{a}$ & $283.88 \mathrm{a}$ & $432.73 \mathrm{a}$ & $722.24 \mathrm{~b}$ & $333.16 \mathrm{a}$ \\
$\mathrm{A} / 22 / 27$ & $61.44 \mathrm{a}$ & $154.02 \mathrm{a}$ & $289.40 \mathrm{a}$ & $430.09 \mathrm{a}$ & $521.80 \mathrm{c}$ & $291.35 \mathrm{a}$ \\
$\mathrm{A} / 22 / 40$ & $62.63 \mathrm{a}$ & $184.38 \mathrm{a}$ & $274.02 \mathrm{a}$ & $442.65 \mathrm{a}$ & $904.12 \mathrm{a}$ & $373.56 \mathrm{a}$ \\
BT1 & $66.90 \mathrm{a}$ & $185.79 \mathrm{a}$ & $319.86 \mathrm{a}$ & $465.94 \mathrm{a}$ & $850.26 \mathrm{a}$ & $377.75 \mathrm{a}$ \\
\hline LSD at 0.05 & NS & NS & NS & NS & 70.80 & 87.04 \\
\hline
\end{tabular}

Within column values followed by different letter ( $\mathrm{s}$ ) are significantly different by DMRT ( $\mathrm{p} \leq 0.05)$

Table-3. Mean yield of green leaf ( $g$ plant $\left.{ }^{-1}\right)$ at mature stage $\left(6^{\text {th }}-14^{\text {th }}\right.$ year)

\begin{tabular}{|c|c|c|c|c|c|c|c|c|c|c|}
\hline \multirow{3}{*}{$\begin{array}{l}\text { Year } \\
\text { Clone }\end{array}$} & tent & 100 & tom & 1. & $0^{\text {th }}$ Year & 11th Yeal & 5 & $3^{\text {th }}$ Year & th Year & \\
\hline & & & & & & & & LSK & LP & \\
\hline & 20 & & 4 & 2005 & & 07 & 2008 & 2009 & 20 & \\
\hline & & & & $941.34 \mathrm{bc}$ & & $804.00 a$ & & $45 \mathrm{~b}$ & $888.1 b$ & \\
\hline & & & & & & & & & & \\
\hline & & & & & & & & & & \\
\hline $22 / 40$ & $759.87 a$ & & 930 & 1 & $.80 a$ & & & $1192.14 \mathrm{a}$ & 1123 & \\
\hline$\Gamma 1$ & $57.46 \mathrm{~b}$ & 48.81ab & 838.83b & 1046.91ab & $745.20 \mathrm{a}$ & 795.66a & $880.89 \mathrm{~b}$ & 1080.72ab & 1003.3ab & $377.53 \mathrm{~b}$ \\
\hline & & & 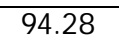 & & & & & 157.96 & 27. & 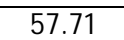 \\
\hline
\end{tabular}
0.05

Within column values followed by different letter ( $\mathrm{s}$ ) are significantly different by DMRT ( $\mathrm{p} \leq \mathrm{0.05})$

Table-4. Estimated made tea yield ( $\left.\mathrm{kg} \mathrm{ha}^{-1}\right)$ at immature stage (1st - $5^{\text {th }}$ year)

\begin{tabular}{lccccc}
\hline \multicolumn{1}{c}{ Year } & $1^{\text {st }}$ Year Skiff & $2^{\text {nd }}$ Year Prune & $3^{\text {rd }}$ Year Skiff & $4^{\text {th }}$ Year Prune & $5^{\text {th }}$ Year Skiff \\
Clone & 1997 & 1998 & 1999 & 2000 & 2001 \\
\hline A/8/01 & 231.45 & 571.35 & 1156.68 & 1443.27 & 2614.12 \\
A/ 17/22 & 211.55 & 616.98 & 1036.39 & 1579.81 & 2636.75 \\
A/22/27 & 224.3 & 562.29 & 1056.54 & 1570.17 & 1904.98 \\
A/ 22/40 & 228.65 & 673.13 & 1000.39 & 1616.02 & 3300.76 \\
BT1 & 244.23 & 678.28 & 1167.74 & 1701.75 & 3104.13 \\
\hline
\end{tabular}

Table-5. Estimated made tea ( $\left.\mathrm{kg} \mathrm{ha}^{-1}\right)$ at mature stage ( $6^{\text {th }}-14^{\text {th }}$ year)

\begin{tabular}{|c|c|c|c|c|c|c|c|c|c|c|}
\hline Year & $\begin{array}{c}6^{\text {th }} \text { Year } \\
\text { LP } \\
2002\end{array}$ & $\begin{array}{c}\text { 7th Year } \\
\text { DSK 200 }\end{array}$ & $\begin{array}{c}8^{\text {th }} \text { Year } \\
\text { MSK } \\
2004\end{array}$ & $\begin{array}{c}9^{\text {th }} \text { Year } \\
\text { LSK } \\
2005\end{array}$ & $\begin{array}{c}10^{\text {th }} \text { Year } \\
\text { LP } \\
2006\end{array}$ & $\begin{array}{c}11^{\text {th }} \text { Year } \\
\text { DSK } \\
2007\end{array}$ & $\begin{array}{c}12^{\text {th }} \text { Year } \\
\text { MSK } \\
2008\end{array}$ & $\begin{array}{c}13^{\text {th }} \text { Year } \\
\text { LSK } \\
2009\end{array}$ & $\begin{array}{c}14^{\text {th }} \text { Year } \\
\text { LP } \\
2010\end{array}$ & Average \\
\hline$\overline{\mathrm{A} / 8 / 01}$ & 2392.11 & 2617.15 & 2725.76 & 3436.64 & 2507.00 & 2935.24 & 2938.89 & 3415.14 & 3242.3 & 2912.24 \\
\hline A/ $17 / 22$ & 2158.50 & 2567.86 & 2666.00 & 3214.60 & 2443.26 & 2882.27 & 2767.22 & 3381.99 & 3292.29 & 2817.76 \\
\hline $\mathrm{A} / 22 / 27$ & 1885.40 & 2076.06 & 1959.42 & 2386.27 & 2551.36 & 2524.85 & 2259.92 & 2542.18 & 2324.10 & 227 \\
\hline $\mathrm{A} / 22 / 40$ & 2774.13 & 3218.62 & 3396.16 & 4201.16 & 2766.57 & 3219.05 & 3550.84 & 4262.49 & 4102.00 & 3509.10 \\
\hline BT1 & 2400.25 & 3098.84 & 3062.40 & 3822.06 & 2720.57 & 2904.79 & 3215.95 & 3945.49 & 3661.8 & 3203.69 \\
\hline
\end{tabular}


At immature stage (1st $-5^{\text {th }}$ year), the highest average made tea yield (1379.08 $\mathrm{kg} \mathrm{ha}^{-1}$ ) was obtained from the standard clone BT1 which was statistically identical with $\mathrm{A} / 22 / 40$ (1364 kg ha-1) (Fig. 1).

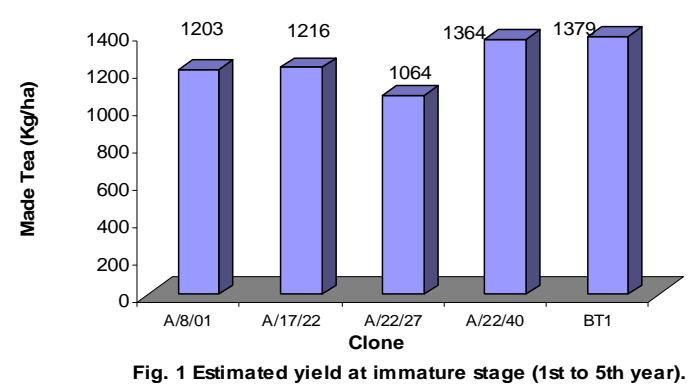

At standard productivity level i.e. after maturity, in the year of $6^{\text {th }}, 7^{\text {th }}, 8^{\text {th }}, 9^{\text {th }}, 12^{\text {th }}, 13^{\text {th }} \& 14^{\text {th }}$ made tea yield variations were significant except in the year of $10^{\text {th }} \& 11^{\text {th }}$. This was occurred due to drought in 2006 and 2007.
On the average of 9 year production, the test clone $\mathrm{A} / 22 / 40$ gave significantly (LSD at 0.05 ) higher yield (3509.1 kg ha-1) followed by A/8/01 (2912.24 kg ha-1), A/17/22 (2817.76 kg ha-1), A/22/27 (2278.97 $\mathrm{kg} \mathrm{ha}^{-1}$ ) over control BT1 (3203.69 kg ha-1) (Fig. 2).

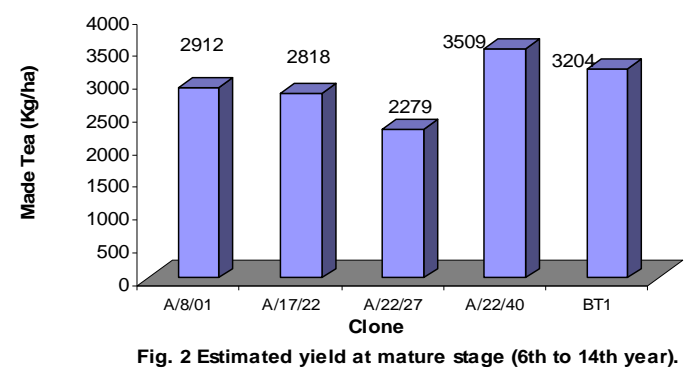

Quality performance: The overall quality performances of the test clones and control are shown in Table 6 . The cup characteristics of all the test clones including the control BT1 were categorized as "Above average" except A/22/27. They have bright infusion, strong liquour colour with useful strength and briskness (Table 6).

Table 6. Cup quality of test clones (Average score of 10 years)

\begin{tabular}{lccccccc}
\hline Test Clone & $\begin{array}{c}\text { Infusion } \\
(10)\end{array}$ & $\begin{array}{c}\text { Liquour } \\
\text { colour }(10)\end{array}$ & $\begin{array}{c}\text { Briskness } \\
(10)\end{array}$ & $\begin{array}{c}\text { Strength } \\
(10)\end{array}$ & $\begin{array}{c}\text { Creaming } \\
\text { down }(10)\end{array}$ & Total & $\begin{array}{c}\text { Overall } \\
\text { Quality }\end{array}$ \\
\hline $\mathrm{A} / 8 / 01$ & $7.23 \mathrm{~b}$ & $7.49 \mathrm{ab}$ & $7.37 \mathrm{~b}$ & $7.14 \mathrm{~b}$ & $3.00 \mathrm{~b}$ & $32.22 \mathrm{bc}$ & $\mathrm{AA}$ \\
$\mathrm{A} / 17 / 22$ & $7.31 \mathrm{~b}$ & $7.61 \mathrm{ab}$ & $7.33 \mathrm{bc}$ & $7.40 \mathrm{a}$ & $3.04 \mathrm{~b}$ & $32.67 \mathrm{~b}$ & $\mathrm{AA}$ \\
$\mathrm{A} / 22 / 27$ & $7.28 \mathrm{~b}$ & $7.46 \mathrm{~b}$ & $7.17 \mathrm{c}$ & $7.14 \mathrm{~b}$ & $2.66 \mathrm{c}$ & $31.74 \mathrm{c}$ & $\mathrm{A}$ \\
$\mathrm{A} / 22 / 40$ & $7.38 \mathrm{~b}$ & $7.56 \mathrm{~b}$ & $7.41 \mathrm{ab}$ & $7.40 \mathrm{a}$ & $3.08 \mathrm{~b}$ & $32.95 \mathrm{ab}$ & $\mathrm{AA}$ \\
$\mathrm{BT} 1$ & $7.54 \mathrm{a}$ & $7.66 \mathrm{a}$ & $7.58 \mathrm{a}$ & $7.41 \mathrm{a}$ & $3.39 \mathrm{a}$ & $33.54 \mathrm{a}$ & $\mathrm{AA}$ \\
\hline LSD at 0.05 & 0.1616 & 0.1786 & 0.1745 & 0.2086 & 0.2409 & 0.7276 & - \\
\hline
\end{tabular}

Within column values followed by different letter ( $\mathrm{s}$ ) are significantly different by DMRT ( $\mathrm{p} \leq \mathrm{0.05})$

$\mathrm{A}=$ Average, $\mathrm{AA}=$ Above Average

Considering the quality standard and yield performances throughout study period, the test clone $\mathrm{A} / 22 / 40$ has appeared superior to the control BT1 and to be released as a standard clone. The other test clone $\mathrm{A} / 8 / 8$ has also found prospective in quality characters and yield, and can be used as a standard clone or valuable breeding stock.

\section{References}

Barua, D.N. 1964. Selection of vegetative clones. Two and a Bud, 11(2): 32-38.

BTB. 2002. Strategic plan for Bangladesh tea industry 2002-2021. Vision 2021. Bangladesh Tea Board, Nasirabad, Chittagong. p. 1.

Dutta, M.J. and Alam, A.F.M.B. 2001. Study the performance of four test clones on the yield and quality of tea. Tea J. Bangladesh, 37 (1\&2): 29-34.

ITC (International Tea Committee). 2011. Annual Bulletin of Statistics 2011. ITC, London, England.
Kibria, A.K.M.G. and Rashid, M.A. 1994. Fertilizer recommendation for mature tea. Pamphlet no. 21. Bangladesh Tea Research Institute, Srimangal, Moulvibazar. pp. 1-20.

Kibria, A.K.M.G. and Uddin, F. 1998. Fertilizer recommendation for young tea. Pamphlet no. 22. Bangladesh Tea Research Institute, Srimangal, Moulvibazar. pp. 1-12.

Njuguna, C.K. 1990. Clonal selection from young tea seedlings in the nursery. Tea Research Global Perspective. pp. 26-31. In: Proc. International Conf. on R \& D in Tea. 11-12 Jan. 1990, Calcutta, India.

Rashid, A. and Alam, A.F.M.B. 1990. Thirty years of clonal selection and breeding at BTRIAchievements and future strategies. Tea Research Global Perspective. pp. 200-206. In: Proc. International Conf. on $\mathrm{R} \& \mathrm{D}$ in Tea. 11-12 J an, 1990, Calcutta, India.

Rashid, S.A. 1986. Mature tea pruning. Circular no. 79. Bangladesh Tea Research Institute, Srimangal, Moulvibazar. pp. 1- 6. 
Shahiduzzaman, M., Sarker, M.L. and Alam, Visser, T. and Kehl, F.H. 1958. Selection and A.F.M.B. 2002. Young tea pruning. Circular. 111. Bangladesh Tea Research Institute, Srimangal, Moulvibazar. pp. 1- 4.

Tubbs, F.R. 1946. Tea selection. Tea Quarterly, 18: 59- 65. vegetative propagation of tea. Tea Quarterly, 29: 76-86.

Wight, N. 1961. Improved methods of clonal selection. Two and a Bud, 8(2): 3-5. 